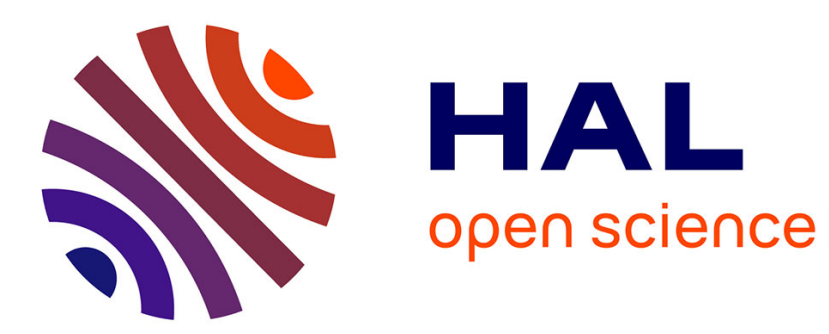

\title{
Nanoscale optical trap for fluorescent nanoparticles
}

Olivier Emile, Janine Emile, Hervé Tabuteau

\section{To cite this version:}

Olivier Emile, Janine Emile, Hervé Tabuteau. Nanoscale optical trap for fluorescent nanoparticles. EPL - Europhysics Letters, 2020, 129 (5), pp.58001. 10.1209/0295-5075/129/58001 . hal-02862822

\section{HAL Id: hal-02862822}

\section{https://hal-univ-rennes1.archives-ouvertes.fr/hal-02862822}

Submitted on 17 Jun 2020

HAL is a multi-disciplinary open access archive for the deposit and dissemination of scientific research documents, whether they are published or not. The documents may come from teaching and research institutions in France or abroad, or from public or private research centers.
L'archive ouverte pluridisciplinaire HAL, est destinée au dépôt et à la diffusion de documents scientifiques de niveau recherche, publiés ou non, émanant des établissements d'enseignement et de recherche français ou étrangers, des laboratoires publics ou privés. 


\title{
Nanoscale optical trap for fluorescent nanoparticles.
}

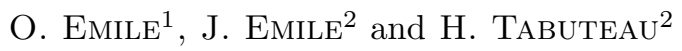 \\ 1 Université de Rennes 1, Campus de Beaulieu, F-35000 Rennes, France \\ 2 Université de Rennes 1, CNRS IPR UMR 6251, F-35000 Rennes, France
}

\author{
PACS 87.80.Cc - Optical trapping \\ PACS 42.50 .Wk - Mechanical effects of light on material media, microstructure and particles in \\ optics \\ PACS 78.45.+h - Stimulated emission
}

\begin{abstract}
Optical trapping has offered new potentialities in the manipulation of bacteria, living cells, organelles, or microparticles in micrometric volumes. Its extension to the nanometer scale may create exceptional opportunities in many areas of science. We report here the trapping of a single 100-nm-radius particle in a nanometric volume. The trapping relies on stimulated emission of the fluorescent particle in the evanescent wave of a totally reflected Arago spot on a glass-liquid interface. The trapping volume is a $200-\mathrm{nm}$-height and $125-\mathrm{nm}$-radius cylinder. Such a nanotrap, aside from applications in nano-physics, may echo the needs of life scientists to manipulate ever-smaller biological samples.
\end{abstract}

Introduction . - The advent of optical tweezers has led to a real revolution in optical manipulation [1] that has spurred a new area of medical, biophysical and physics discovery. This non-contact manipulation technique enables mechanical force measurements [2,3], 3D micropositioning [4], micro-fabrication assembly [1,5], cell sorting [6], tissue engineering [7], to name a few. Nevertheless, direct manipulation of matter at the nanometer scale remains a significant challenge for optical tweezers [8], because, on the one hand, the traps are micrometer size limited [9], and on the other hand, because the trapping force diminishes as the third power of the particle diameter [10-12].

Two main directions have been followed to circumvent these limitations [13]. The first one combines tight light focusing and total internal reflection [14-18]. However, whereas the trap size is then in the nanometer range in the direction of the evanescent wave, it is still limited by diffraction in the other directions. The other one uses plasmon enhanced electromagnetic field to break the diffraction limit [19-23]. This can be realized for example, at the end of an optical fibre $[24,25]$. Alternatively, polystyrene nano-spheres have been trapped on nanostructured substrates or plasmonic nanopores [26], as well as molecules of biological interest [27], or metallic particles [12]. Nevertheless, these nanometric optical tweezers are based on dedicated plasmonic structures. Versatile nanometer size optical traps are still lacking.
Experimental set-up. - In order to address this challenge, we propose to trap nanoparticles using an Arago spot [28] (Fig. 1a) that experiences total internal reflection at a glass-liquid interface. Whereas Gaussian beams are diffraction limited to a fraction of wavelength, such Arago spots can have smaller sizes. The intensity distribution can be written as [29]

$$
I(r)=I_{0} \frac{h^{2}}{h^{2}+(d / 2)^{2}} J_{0}^{2}\left(\frac{\pi r d}{\lambda\left(h^{2}+(d / 2)^{2}\right)^{1 / 2}}\right)
$$

$J_{0}$ being the zeroth order Bessel function of the first kind, $h$ the distance between the disk and the glass-liquid interface ( $h$ equals the thickness of a BK7 glass lamella, $h=170 \mu \mathrm{m}), r$ the distance to the beam axis, $\lambda$ the light wavelength, $d$ the disk diameter (see Fig. 1a) and $I_{0}$ the intensity of the incoming light at the edges of the disk. The maximum light intensity of the spot at the interface is of the order of $I(r=0)=100 \mathrm{~mW} \cdot \mathrm{mm}^{-2}$. We characterize its size by its waist $w_{0}$, as for Gaussian beams. It is the radius at which the intensity values fall to $1 / e^{2}$ of its axial value. It here equals to $w_{0}=120 \mathrm{~nm}$, which would be half of the waist of a Gaussian beam of the same wavelength, focused by a lens of numerical aperture equal to 1 . However, in the case of a Gaussian beam, there is a large spreading of the direction of the wavevector, whereas, in our case, the angle between the direction of propagation and the wavevectors is constant. The wavevectors lie on a cone. 


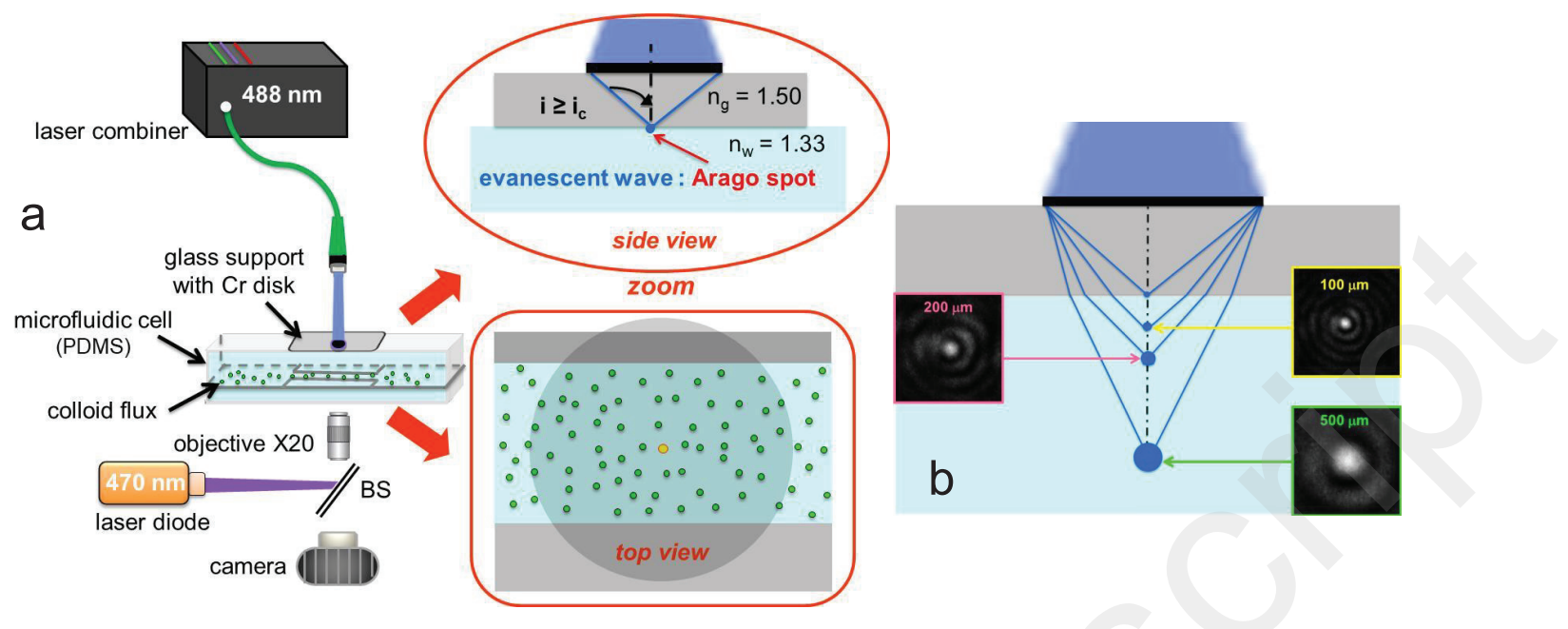

Fig. 1: a) Fluorescent particles (Molecular Probes, diameter $2 a=200 \mathrm{~nm}$ ) suspended in water, flowing in a $30 \mu \mathrm{m}-\mathrm{height}, 300$ $\mu$ m-width PDMS (PolyDiMethilSiloxane) micro-channel made by standard soft lithography [30] covered with a glass lamella. The flow can be varied and controlled with a microfluidic pump. The particles are excited from underneath with an optical diode (ThorLabs M470L2, $470 \mathrm{~nm}$ central wavelength, power $700 \mathrm{~mW}$, focused to a $1 \mathrm{~mm}$ diameter spot on the channel), and trapped in an Arago spot laser originating from the interference of a collimated laser beam (L4Cc Oxxius, wavelength $\lambda=488$ $\mathrm{nm}$, power $250 \mathrm{~mW}$ at the end of an optical fibre) diffracted by the edges of a $d=650 \mu \mathrm{m}$ chromium occulting disk deposited on a glass substrate. We use an inverted microscope (Leica, X20 objective lens, NA 0.4) equipped with a motorized XY stage. Pictures are taken every $30 \mathrm{~ms}$ by a SCMOS monochrome gray-scale camera (Orca flash 4.0 Hamamatsu) and analyzed using ImageJ software [31]. $i$ : angle of incidence, $i_{c}$ : critical angle. b) Propagation of the Arago spot in the liquid medium that enables the localization of the spot.

In order for the Arago spot to be under total internal reflection conditions, the angle of incidence $i$ of the diffracted light by the disk $\left(i=\tan ^{-1}(d / 2 h)=62.39^{\circ}\right)$ is higher than the critical angle $i_{c}=\sin ^{-1}\left(n_{w} / n_{g}\right)=61.74^{\circ}$, $n_{w}=1.33$ and $n_{g}=1.51$ being the optical index of water and glass, respectively. The fluorescent particles consist of $N=1.1 \times 10^{5}$ fluorescein molecules (lifetime $\tau_{f}=4.1 \mathrm{~ns}$ ), leading to a fluorescent duty cycle (absorption followed by a spontaneous emission process) of $\Gamma=(N s) /\left(\tau_{f}(1+s)\right) \simeq 2 \times 10^{13} \mathrm{~s}^{-1}$ with a saturation of the dye of $s=2$ estimated from the experimental laser intensity.

The transmitted rays with angle of incidence smaller than $i_{c}$, form an Arago spot in the lower index medium. However, it is not so easy to find the trap location experimentally. To determine the position of the totally reflected Arago spot on the glass-liquid interface, we first image the spot using a $2 \mathrm{~mW}$ laser beam at several distances below the interface (500 $\mu \mathrm{m}$ to $100 \mu \mathrm{m}$, see Fig.1b), where the spot size is in the tens micrometer range. Then, by extrapolation, we define a $10 \mu \mathrm{m}$-diameter area on the interface where we expect the total reflection of the Arago spot to occur. We concentrate the investigation of particles in this area only.

The rays diffracted by the disk impinge on the glassliquid interface under different conditions. Close to the Arago spot, we have identified three types of rays: i) those impinging with an angle of incidence $i$ higher than (but very close to) the critical angle $i_{c}$ (Fig. 2a). They contribute mostly to the trapping mechanism. ii) Those with $i>i_{c}$ (Fig. 2b) which also participate in the trapping but with a lesser extent. iii) Those with $i<i_{c}$ which do not participate in the trapping (Fig. 2c).

Trapping mechanism. - Let us have a closer look at the reflection of these rays and at the electromagnetic field close to the Arago spot. In Fig. 2a, the light rays lead to an Arago spot on the glass-liquid interface. These rays are totally reflected on the interface. They generate an evanescent wave running away from the spot. The evanescent wave exists on a distance corresponding to the so-called Goos-Hänchen shift $\delta_{G H}[32]$. In Fig. 2b, the Arago spot is above the glass-liquid interface. The associated rays are also under total reflection condition. The evanescent wave lasts on a smaller distance since $\delta_{G H}$ is smaller (the angle of incidence is higher). It forms a kind of donut centered on the Arago spot. In Fig. 2c, the rays are not totally reflected. However, because of the laws of refraction close to the critical angle, the transmission coefficient is very low and only a very little part of the light is transmitted [28]. Most of the rays are reflected with no evanescence. Those rays hardly contribute to the electromagnetic field in the liquid medium, close to the interface.

Consequently, on the glass-liquid interface, in the liquid medium, the electric field is composed of outgoing evanescent waves centered on the Arago spot. Similar donut 
Nanoscale optical trap for fluorescent nanoparticles.

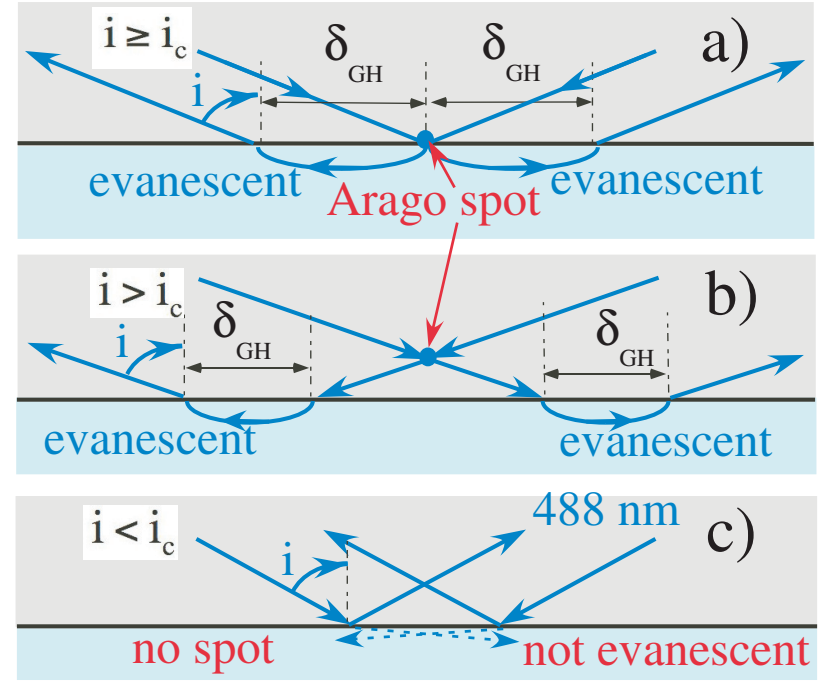

Fig. 2: Electromagnetic field at the glass-liquid interface near the Arago spot. Rays diffracted from the disk: a) that form a spot at the interface (angle of incidence $i$ close to $i_{c}, \delta_{G H}$ : Goos-Hänchen shift); b) that form a spot above the interface ( $i$ higher than $i_{c}$ ), in a) and $\mathrm{b}$ ), rays are totally reflected and generate outgoing evanescent waves; c) impinging on the interface with an angle smaller than $i_{c}$ (they are partially reflected, with no evanescent wave). The doted arrows correspond to the transmitted beam.

shape evanescent waves can be obtained using an occulted beam focused by a high numerical objective [15]. However, in that experiment, the optical beam results from a focusing and its size is thus limited by diffraction. It corresponds to a size of the order of a wavelength, whereas in our experiment, the beam size is smaller.

Nevertheless, the 470-nm light also plays a role. It is responsible for the trapping in the vertical direction. Each fluorescent particle within the flow interacts with this field. In a fluorescent cycle, the particle absorbs and gets excited. Since light carries linear momentum, due to momentum conservation, the particle recoils towards the glass-liquid interface. The radiation pressure force is estimated to $F_{R}=n_{w} \hbar k \Gamma=3.3 \times 10^{-14} \mathrm{~N}$, where $\hbar$ is the reduced Planck constant and $k$ is the wavevector. It acts on all the particles all along the channel, which therefore flow just underneath the glass-liquid interface. The electrostatic repulsion prevents them to stick to the glass, and keeps them away at a fraction of hundreds of nanometer distance [33]. Thereafter, particles fluoresce and recoil in a random direction.

What is then the trapping mechanism in the horizontal plane? It couldn't be via gradient forces. Compared with the previous experiments that trap particles in 3D using evanescent waves [15-17], our particles are 10 times smaller and the light intensity of the Arago spot is $10^{-5}$ times smaller. Then, the gradient force falls below $10^{-21}$ N. Actually, when the particle is close to the Arago spot, the $470 \mathrm{~nm}$-light still enlightens it. After excitation, it can either fluoresce as described before, or emit a stimulated photon in the evanescent wave at $488 \mathrm{~nm}$ (Fig. 3). This photon has exactly the same characteristics as the photons in the evanescent wave. The photon momentum is thus always directed outward from the spot (Fig. 2a and $2 \mathrm{~b}$ ). Because of linear momentum conservation, the particle is pushed towards the spot (Fig. 3a and 3c). This leads to a trapping force in the horizontal plane, with a capture range of about $r_{t}=125 \mathrm{~nm}$. At the Arago spot position, the force cancels (Fig. 3b).

Small particles have already been trapped using focused super-oscillating beams [34]. However, the trapping mechanism they described is based on the dipolar force that strongly decreases when decreasing the trapped particle size. Besides, in this super-oscillating beam, a side lobe emerges very close to the fundamental lobe. This side lobe is much more intense than the fundamental one. Then, for very small beams, particles may be trapped in the side lobe instead of the fundamental one. That may be detrimental to reach small trap sizes. Moreover, whereas their trapping mechanism is very efficient in $2 \mathrm{D}$, it is less efficient in the direction of the focusing of the beam, with a trapping size in the micrometer range as for optical tweezers. This is not the case of our trap where the trapping volume is the same in the three dimensions.

Results. - An example of a single trapped particle is shown in Fig. 4 (Fig. 4e to 4i). We fit the fluorescent signal of the particle with a Gaussian distribution intensity, and we assimilate the centre of the Gaussian with the centre of the particle [35], with $15 \mathrm{~nm}$ precision. Such a trajectory appears in Fig. $4 \mathrm{~m}$ and $4 \mathrm{n}$. During the trapping, the particle exhibits a Brownian like motion inscribed within a circle corresponding to the size of the trap. The position of the particle within the trap doesn't reflect the trapping potential of the light that is supposed to be harmonic. We will focus on the motion of the particle in the trap below (see end of following section). The trapping volume thus consists of a-125-nm radius cylinder in the horizontal plane whose height is limited by the penetration depth of the evanescent wave, i.e. about 200 $\mathrm{nm}$.

In order to check whether the particle could be trapped by the gradient force instead of the stimulated emission force, we have performed the following experiment. We have replaced the $488 \mathrm{~nm}$ laser by a $404 \mathrm{~nm}$ laser. The fluorescein molecules cannot emit a photon in a stimulated way at this wavelength. However, for the same laser power, the gradient force is unchanged. We haven't noticed any particle trapping. This reinforces the stimulated emission hypothesis.

\section{Discussion. -}

Magnitude of the force. Let us evaluate the magnitude of the trapping force. To this purpose, we have to estimate the number of stimulated photons involved in the experiment. This can be performed via evolution equations [36]. 


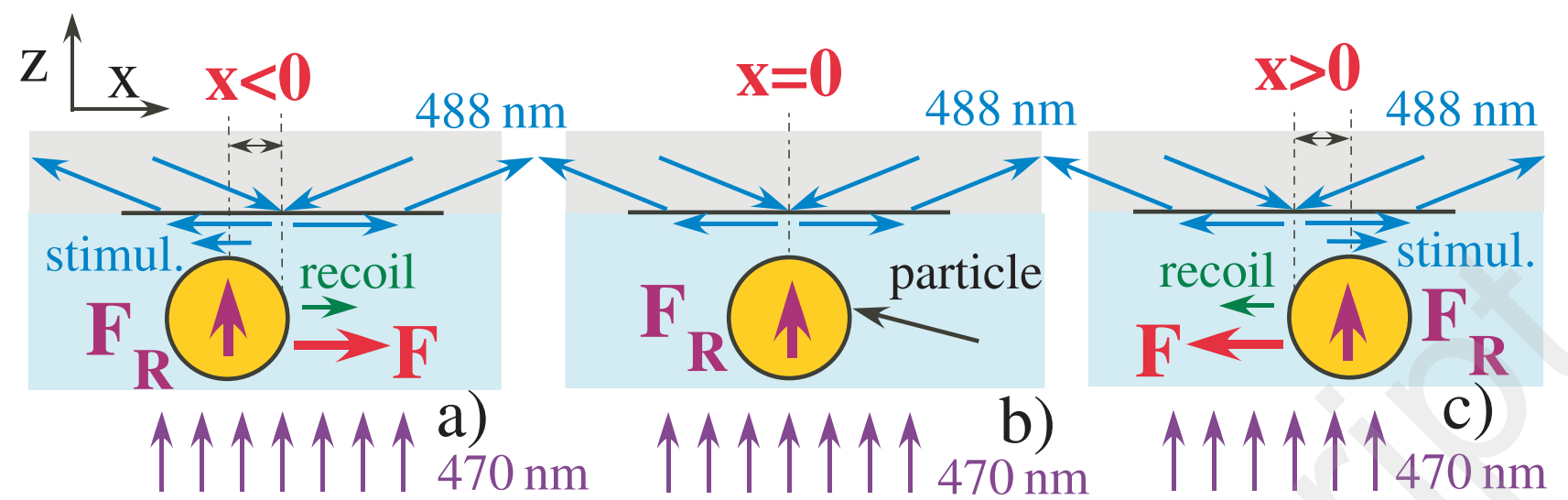

Fig. 3: Trapping mechanism. The radiation pressure force $F_{R}$ from the $470 \mathrm{~nm}$ light (vertical arrows), pushes the particle in the upward direction, leading to a vertical trapping. In the horizontal plane, a) at a distance $x<0$ from the spot, the excited particle, emits light in a stimulated way in the evanescent wave that goes out from the spot (horizontal arrows). Due to momentum conservation, the particle recoils towards the spot, leading to a spring force $F$. b) At the spot location $x=0$, stimulated emission in both directions compensates. There is no resultant force. c) When $x>0$, the effect is reversed compared to a). The particle is pushed towards $x=0$.
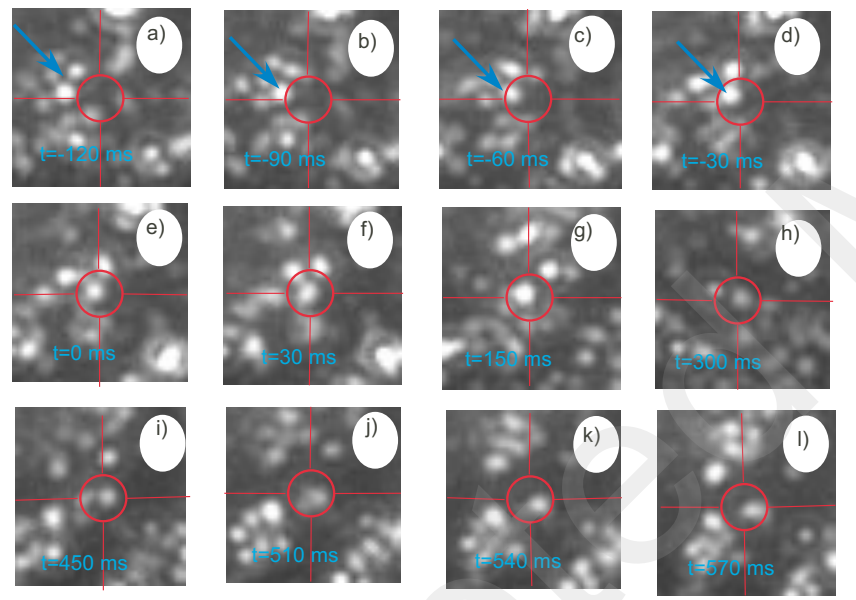

n)

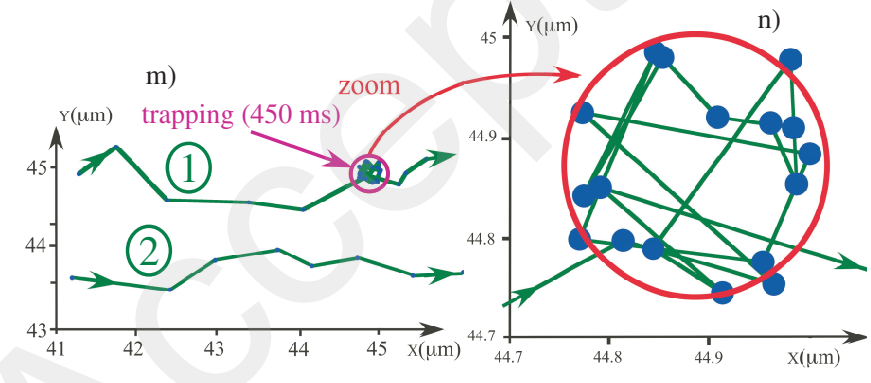

Fig. 4: Pictures of a trapped particle and particle trajectory. a) to d) Particle before trapping. The blue arrows point toward the particle. e) to i) Trapped particle (trapping period $450 \mathrm{~ms}$ ). j) to 1 ), particle escape. The red $3 \mu$ m-diameter circle highlights the trapping zone (much larger than the trap). m) Trajectory of a trapped particle before, during and after trapping (label 1). Trajectory of a non-trapped particle (label 2). n) Zoom of the particle trajectory during trapping. The diameter of the purple circle corresponds to the horizontal trap dimension.
Experimentally, we observe a decrease of the fluorescence of the trapped particle by about $2 / 3$ compared with the non-trapping period. It then recovers its initial fluorescent rate when leaving the trap, in a way similar to the selective deactivation of fluorophores in STED microscopy [37]. In order to explain this decrease, let us write a simplified detailed balancing at thermal equilibrium of the absorption and emission process [38] in a cycle,

$$
A n_{e}+B n_{e} \rho(\nu)-B n_{g} \rho\left(\nu_{0}\right)+C n_{e}=0
$$

where $A$ and $B$ are the so-called Einstein coefficients, $n_{g}$ and $n_{e}$ are the populations of the ground and excited states of the particle $\left(n_{g}+n_{e}=1\right)$, respectively, and $\rho\left(\nu_{0}\right)$ and $\rho(\nu)$ are the spectral density energies of light at 470 $\mathrm{nm}$ and at $488 \mathrm{~nm}$, respectively. $C n_{e}$ corresponds to non radiative decay from the excited state. The first term in Eq. $2\left(A n_{e}\right)$ accounts for the spontaneous emission, the second one $\left(B n_{e} \rho(\nu)\right)$ accounts for the stimulated emission at $488 \mathrm{~nm}$, and the third term $\left(B n_{g} \rho\left(\nu_{0}\right)\right)$ accounts for the absorption of light at $470 \mathrm{~nm}$. In the absence of the Arago spot, Eq. 2 leads to $A n_{e}^{0}-B n_{g}^{0} \rho\left(\nu_{0}\right)+C n_{e}^{0}$. The superscript accounts for the populations without the Arago spot.

Let us call $\delta$ the ratio between the spectral energy densities at $488 \mathrm{~nm}$ and at $470 \mathrm{~nm}\left(\delta=\rho(\nu) / \rho\left(\nu_{0}\right)\right)$, and $\alpha$ the decrease of the fluorescence due to the spot $\alpha=n_{e} / n_{e}^{0}$. Straightforward calculations lead to $n_{e}^{0}=(1-\alpha) /(\alpha \delta)$. In our experiment, $s=2, n_{e}^{0}=2 / 3$. The light intensity at $470 \mathrm{~nm}$ and at $488 \mathrm{~nm}$ are nearly the same $(\delta=1)$, leading to $\alpha=3 / 5$. This value of $\alpha$ is about the value we found experimentally. Using this value of $\alpha$, we can estimate the force due to stimulated emission. Indeed, pushing forwards the calculations, we find $B n_{e} \rho(\nu)=6 / 5 B n_{e}^{0} \rho\left(\nu_{0}\right)$, leading to $F=6 / 5 F_{R}=4 \times 10^{-14} \mathrm{~N}$.

This value of the force can be checked experimentally. 
The mean value of the square of the position of a trapped particle evaluated from Fig. $4 \mathrm{n}$ is $\sqrt{\left\langle x^{2}\right\rangle} \sim 10^{-7} \mathrm{~m}$, assuming an harmonic potential. We estimate the temperature of the particle to be around $T=323 \mathrm{~K}$ due to heating, leading to a thermal agitation $k_{B} T=4.5 \times 10^{-21} \mathrm{~J}, k_{B}$ being the Boltzmann constant. The equipartition theorem leads to a trap stiffness $\kappa=k_{B} T /\left\langle x^{2}\right\rangle \sim 4 \times 10^{-7}$ N.m ${ }^{-1}$ and a force $F \sim \kappa \sqrt{\left\langle x^{2}\right\rangle} \sim 4 \times 10^{-14}$ N. This experimentally deduced value of the force thus validates the calculation of the trapping force using Eq. 2.

Trap lifetime. We have followed the trapping of 160 particles (Fig. 5). The trapping time depends on the flow velocity: the slower the flow velocity, the longer the trap lasts. To estimate the trap lifetime $\tau$, several quantities have to be considered. The first one is the momentum diffusion [39], due to the random recoil following an absorption-spontaneous emission cycle. The momentum diffusion coefficient equals to $D=\hbar^{2} k^{2} \Gamma=5 \times 10^{-41}$ J.kg. $\mathrm{s}^{-1}$. Note that this momentum diffusion coefficient is different from the usual position diffusion coefficient defined by Einstein $D_{e}=k_{B} T / \gamma, \gamma=6 \pi a \mu$ being the friction coefficient and $\mu$ the dynamical viscosity. Indeed, the Einstein coefficient is relevant for a diffusion process in position, whereas, in our case, in order to estimate the trapping time, we have to consider the diffusion in the momentum space.

The second relevant quantity for the trap lifetime is the depth of the trapping potential $U_{0}$. It equals the force $F$ times the capture range $\left(r_{t}=125 \mathrm{~nm}\right)$, leading to $U_{0}=5 \times$ $10^{-21} \mathrm{~J}$. The third term is the thermal agitation $\left(k_{B} T=\right.$ $\left.4.5 \times 10^{-21} \mathrm{~J}\right)$. Note that we consider the trapping in the horizontal plane only, the vertical trapping is due to the radiation pressure as already explained. The last term accounts for the friction with the flow ( $v$ being the flow velocity). The following equation then sets

$$
\frac{1}{m} D \tau=U_{0}-k_{B} T-\gamma v r_{t}
$$

At $323 \mathrm{~K}, \mu=0.53 \times 10^{-3}$ Pa.s. One finds $\gamma r_{t}=1.2 \times$ $10^{-16}$ N.s. The damping ratio is $\gamma /(2 \sqrt{m \kappa})=400$. One expects a linear variation of $\tau$ versus the flow velocity, which is indeed experimentally observed (Fig. 5b).

From this figure, the maximum trapping time is $\tau=0.8$ s leading to a diffusion term $D \tau / m=10^{-23} \mathrm{~J}$, whereas $U_{0}-k_{B} T=5 \times 10^{-22} \mathrm{~J}$. This more than one order of magnitude discrepancy could be explained by the following arguments: i) the potential $U_{0}$ and the thermal agitation are of the same order of magnitude. A variation of $8 \%$ of the trapping force $F$ would lead to a decrease of $U_{0}$ $k_{B} T$ by a factor of 5 . ii) The spontaneous photon may undergo amplified spontaneous emission [40]. Then the step of the random walk in momentum space is multiplied by a number corresponding to the number of stimulated photons, leading to a dramatic increase of $D$. For example, 3 amplified spontaneous photons would lead to an increase of $(3+1)^{2}=16$ in the diffusion coefficient.
From Fig. 5, $v=45 \mu \mathrm{m} . \mathrm{s}^{-1}$ corresponds to $\tau=0$. The friction term then equals $\gamma v r_{t}=5 \times 10^{-21} \mathrm{~J}$. This is one order of magnitude higher than the other terms in Eq. 3. It has to be noted that this friction term is relative to the flow around the particle ( $v$ is the flow velocity). However, this flow velocity $v$ is estimated from the fluorescent particles around the trapped particle. The trapped particle is close to the interface, at an estimated distance of 100 nm, whereas the non-trapped particles are at much higher distances. For example, in a Poiseuille flow of radius 15 $\mu \mathrm{m}$, the velocity from $1.5 \mu \mathrm{m}$ to $100 \mathrm{~nm}$ decreases by a factor of 10 . The flow velocity in Fig. 5 is thus overestimated. Then, the experimental results agree with the model described by Eq. 3.

In order for the trapping to be efficient, the trapping potential should be around 10 times the thermal agitation [41]. In our experiment, the trapping potential is of the order of the thermal agitation, limiting the trap lifetime to $0.8 \mathrm{~s}$ at most (Fig. 5b). During this time, a laser pulse could be sent also via the optical fiber in order to induce a chemical reaction for the trapped particle. The particle would be glued to the substrate. Then, thanks to the microstage, the spot could be moved, another particle could be trapped. One can thus print a regular pattern of glued particles on the substrate.

Nevertheless, this trapping time is too low for the usual applications of optical traps. However, an increase of the $488 \mathrm{~nm}$ laser power by a factor of 10 would increase the intensity of the evanescent wave by a factor of 10 without reaching the damage threshold of the particles. The stimulated emission and thus the trapping force in the horizontal direction would increase accordingly. Besides, a greater $488 \mathrm{~nm}$ laser power will increase the stimulation emission process and thus reduces the population of the excited state of the fluorescent particles. It will thereby increase the radiation pressure force and the trapping in the vertical direction, and will lower the momentum diffusion. This would boost the trapping time to the hundreds of seconds range.

The motion of the particle inside the trap still needs to be addressed (see Fig. 4n). This motion may be due to temperature gradients [42] and self thermophoresis. Actually, For a trapped particle, in an absorption (mean wavelength $\lambda_{1}=470 \mathrm{~nm}$ ) spontaneous emission (mean wavelength $\lambda_{2}=550 \mathrm{~nm}$ ) cycle, the energy difference between the two wavelengths is $\Delta E=h c\left(1 / \lambda_{1}-1 / \lambda_{2}\right)=$ $6.1 \times 10^{-20} \mathrm{~J}, c$ being the light velocity. The number of cycles for trapped particles equals $\Gamma / 3$ (see the paragraph about the discussion of the force). The power to be evacuated is of the order of $P=\Delta E \times \Gamma / 3 \simeq 4 \times 10^{-7} \mathrm{~W}$. The trapping region radius is $r_{t}=125 \mathrm{~nm}$. The heat flux equation [43] across the surface limiting the trapping volume ( $S=4 \pi r_{t}^{2}$, spherical symmetry), assuming a steady state flux for a mean particle position at the center of the trap, leads to a temperature gradient $\frac{\partial T}{\partial r}=\frac{P}{S k} \simeq 3 \times 10^{6} \mathrm{~K} . \mathrm{m}^{-1}$, $k=0.6 \mathrm{~W} \cdot \mathrm{m}^{-1} \cdot \mathrm{K}^{-1}$ being the thermal conductivity of water and $r$ the radial distance. The drift velocity [44] is then 

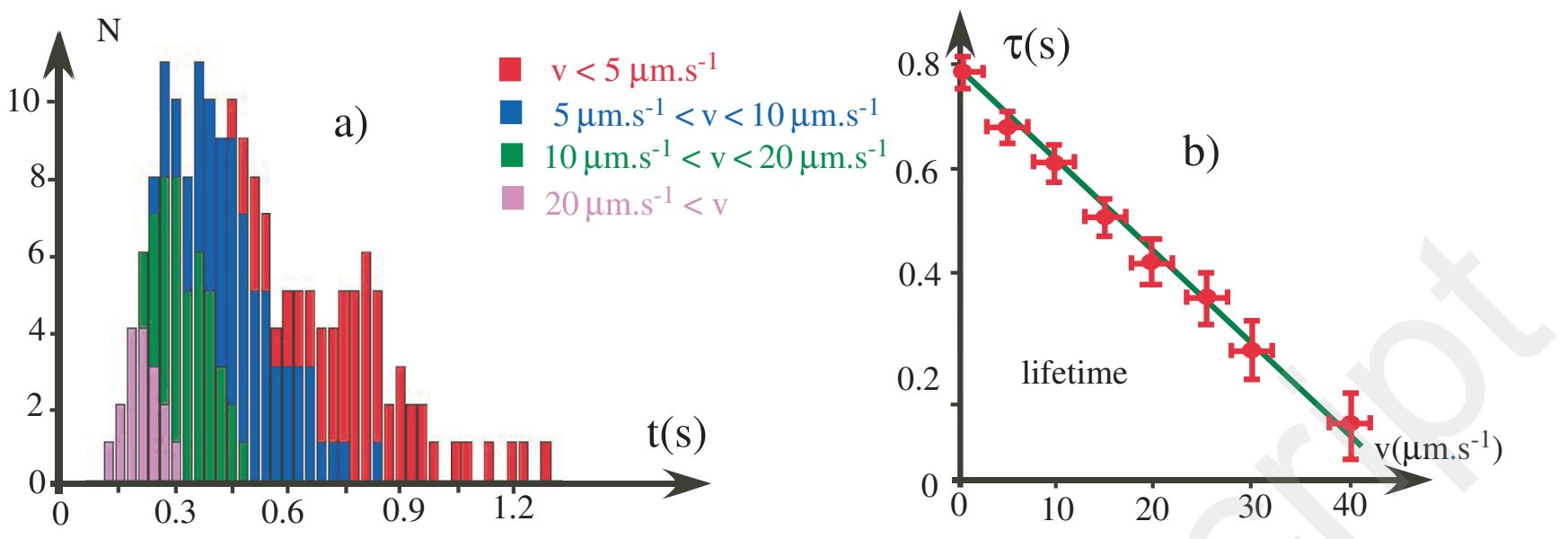

Fig. 5: Trap lifetime. a) Histogram of the trapping period for different flow velocities. $N$ : number of particles that have been trapped. b) Variation of the mean trapping lifetime versus flow velocities. Red crosses: experimental data (the size of the cross represents the measurement uncertainty), green line: linear adjustment.

$u=D_{T} \frac{\partial T}{\partial r} \simeq 3 \mu \mathrm{m} \cdot \mathrm{s}^{-1}, D_{T}=10^{-8} \mathrm{~cm}^{2} \cdot \mathrm{s}^{-1} \cdot \mathrm{K}^{-1}$ being the thermophoretic mobility. This drift velocity decreases as the particle reaches the edges of the trap.

The order of the magnitude of the velocity drift in the vicinity of the center of the trap is in the $\mu \mathrm{m} . \mathrm{s}^{-1}$ range, i.e., of the same magnitude as the flow velocity. This may prevent the particle to stay in the trap center. The particle seems to be located at the edges of the trap (see Fig. 4n). Although the radius of the trap is very small, this drift is one drawback of using fluorescent particles. This may also be a limiting factor of the measured lifetime. It is worth noting that similar position distributions have been encountered in the trapping of fluorescent particles, out of equilibrium $[45,46]$. However these particles were Janus particles and the direction of the thermophoretic force is associated with their dissymmetry. The explanation in our case is different since we deal with spherical particles.

The trapped particles are $100 \mathrm{~nm}$ radius fluorescent particles. Since the trapping mechanism relies on stimulated emission, the magnitude of the force only depends on the fluorescent duty cycle $\Gamma$. This mechanism can be transposed to smaller particles (in the 10 of nanometer range or below). The number of fluorescent cycles would of course diminished, but the mass of the particle would also diminish accordingly. The trapping efficiency would remain the same, whatever the size or the particle material. The only constraint is that the particles must absorb and reemit light.

Conclusion. - To conclude, we have described a new way to capture and trap fluorescent nanoparticles flowing inside a liquid. Compared with other techniques using gradient forces, the laser intensity is rather low (in the W.cm ${ }^{-2}$ range). This enables the manipulation of nano-objects inside living organisms below photodamage. Besides, the trap position can be moved by sliding the occulting disk on the channel, in a way analogous to the move of an immersion objective in a microscope. Although the experiment has been performed with blue lasers, it could be easily transposed to any wavelength, including infrared, depending on the transparency window of the experiment, just by changing the fluorescent particles. For example, such trapping system in the surface-plane could be used to trap fluorophores attached to organelles, viruses or even proteins in a lab on-a-chip integration. The size of conventional optical tweezers is too big to trap them efficiently. Such nanometer scale traps could then serve in transfection and single-and sub-cellular surgery [47] or for single-cell biopsies [48].

Conversely, looking at even smaller scales, the trapping mechanism evoked here, which is borrowed from laser trapping techniques [49], could trap several atoms or even molecules in a very small volume. In this later case, the experiment would hold in vacuum and the optical transitions of the atoms or molecules would replace the transition of the dye molecules in the particle. It could thus be an alternative route towards Bose-Einstein condensation [50] using a smaller number of atoms or molecules in a much smaller volume. Applications in the transport of cold atoms could also be considered [51]. Besides the tight confinement of the evanescent wave near the interface may open new opportunities in the study of non-linear optics in atomic media [52].

We acknowledge support from the Contrat Plan Etat Région CPER BUFFON. We would also like to thank an anonymous referee for its suggestions about the selfthermophoresis effect.

\section{REFERENCES}

[1] Grier D. G., Nature, 424 (2003) 810. 
[2] Kishino A. and Yanagida T., Nature, 334 (1998) 74.

[3] Ether D. S. et al, EPL, 112 (2015) 44001.

[4] Capitanio M. and Pavone S., Biophys. J., 69 (2013) 1293.

[5] Maragò O. M., Jones P. H., Gucciardi P. G., Volpe G. and Ferrari A. C., Nat. Nanotechnol., 8 (2013) 807.

[6] Wang X., Chen S., Kong M., Wang Z., Costa K. D., Li R. A. and Sun D., Lab Chip, 11 (2011) 3656.

[7] Kirkham G. R. et al., Sci. Rep., 5 (2015) 8577.

[8] Choudhary D., Mossa A., Jadhav M. and Cecconi C., Biomolecules, 9 (2019) 23.

[9] Daly M., Sergides M. and Chormaic N. S., Laser Photon. Rev., 9 (2015) 309.

[10] Maia Neto P. A. and Nussenzweig H. M., Europhys. Lett., 50 (2000) 702.

[11] Spesyvtseva S. E. S. and Dholakia K., ACS Photonics, 3 (2016) 719.

[12] Melzer J. E. and McLeod E., ACS Nano, 12 (2018) 12440 .

[13] Neuman K. C. and Block S. M., Rev. Sci. Instrum., 75 (2004) 2787.

[14] Almans E. and Brevik I., J. Opt. Soc. Am. B, 12 (1995) 2429.

[15] Gu M., Haumonte J.-B., Micheau Y., Chon J. W. N. and Gan X., Appl. Phys. Lett., 84 (2004) 4236.

[16] Ganic D., Gan X. and Gu M., Opt. Express, 12 (2004) 5533.

[17] Yoon Y. Z. and Cicuta P., Opt. Express, 18 (2010) 7076 .

[18] Emile O., Emile J. and Tabuteau H., EPL, 116 (2017) 64003 .

[19] Novotny L., Bian R. X. and Xie S., Phys. Rev. Lett., 79 (1997) 645.

[20] Volpe G., Quidant R., Badenes G. and Petrov D., Phys. Rev. Lett., 96 (2006) 238101.

[21] Juan M. L., Righini M. and Quidant R., Nat. Photonics, 5 (2011) 349.

[22] Crozier K. B., Light Sci. Appl., 8 (2019) 35.

[23] Kotsifaki D. G. and Chormaic S. N., Nanophot., 8 (2019) 1227.

[24] Klar T., Perner M., Grosse S., Von Plessen G. Spirkl W. and Feldmann J., Phys. Rev. Lett., 80 (1998) 4229.

[25] Tang X., Zhang Y., Su W., Zhang Y., Liu Z., Yang X., Zhang J., YANG Y. and YuAN Y., Opt. Lett., 44 (2019) 5165.

[26] Grigorenko A. N., Roberts N. W., Dickinson M. R. and Zhang Y., Nat. Photonics, 2 (2008) 365.

[27] Galloway C. M., Kreuzer M. P., Ac̀movic̀ S. S., Volpe G., Correia M., Petersen S. B., NevesPetersen M. T. and Quidant R., Nano Lett., 13 (2013) 4299.

[28] Hecht E., Optics (Addison-Wesley, San Fransisco) 2001.

[29] Emile O. and Emile J., Appl. Opt., 59 (2020) 1678.

[30] Squires T. M. and Quake R. S., Rev. Mod. Phys., 77 (2005) 977.

[31] Schneider C. A., Rasband W. S. and Eliceiri K. W., Nat. Methods, 9 (2012) 671.

[32] Le Floch A., Emile O., Ropars G. and Agrawal G., Sci. Rep., 7 (2017) 9083.

[33] ILER, The Chemistry of Silica (Wiley, New York) 1979.

[34] Singh B. K., Nagar H., Roichman Y. and Arie A.,
Light Sci. Appl., 6 (2017) e17050.

[35] Yildiz A., Forkey J. N., McKinney S. A., HA T., Goldman Y. E. and Selvin P. R., Science, 300 (2003) 2061.

[36] Verdeyen J. T., Laser electronics, $3^{\text {rd }}$ Ed. (Prentice Hall, New York) 2001.

[37] Hell S. W. and Wichmann J., Opt. Lett., 19 (1994) 780 .

[38] Einstein A., Verh. Deutsch. Phys Gesell., 18 (1916) 318.

[39] Cohen-Tannoudji C., Dupont-Roc J. and GrynberG G., Atom-Photon Interactions, Basic Processes and Applications (Wiley-VCH) 1998.

[40] Demchenko A. P., Introduction to fluorescence sensing (Springer Science + Business Media BV) 2008.

[41] Ashrin A., Dziedzic J. M., Buorkholm J. E. and Chu S., Opt. Lett, 11 (1986) 288.

[42] Braun D. and Libchaber A., Phys. Rev. Lett., 89 (2002) 188103.

[43] Sears F. W., Young H. D. and Zemansky M.W., University Physics nth $^{\text {Ed }}$. (Reading M. A., Addison-Wesley) 1987.

[44] Braibanti M., Vigolo D. and Piazza R., Phys. Rev. Lett., 100 (2008) 108303.

[45] Moyses H., Palacci J., Sacanna S. and Grier D.G., Soft Matter, 12 (2016) 6357.

[46] Shen C. and Ou-Yang H. D., Proc. SPIE, Optical Trapping and Optical Micromanipulation XVI, 11083 (2019)

[47] Villangca M., Casey D. and Glückstad J., Biophys. Rev., 7 (2015) 379.

[48] Nadappuram B. P., et al., Nat. Nanotechnol., 14 (2019) 80.

[49] Phillips W. D., Rev. Mod. Phys., 70 (1998) 721.

[50] Burt E. A., Ghrist R. W., Myatt C. J., Holland M. J., Cornell E. A. and Wieman C. E., Phys. Rev. Lett. , 79 (1998) 337.

[51] Couvert A., Kavalec T., Reinaudi G. and GueryOdELIN D., EPL, 83 (2015) 13001.

[52] Kumar R., Gokhroo V., Deasy K. and Chormaic S. N., Phys Rev. A, 91 (2015) 053842. 